(C) 2002 Springer-Verlag. Reprinted with permission from Wood Science and Technology 36, number 2, pages 101-110.

Wood Science and Technology 36 (2002) 101-110 (C) Springer-Verlag 2002

DOI 10.1007/s00226-001-0137-x

\title{
Originals
}

\section{Structural features of water-soluble arabinogalactans from Norway spruce and Scots pine heartwood}

\author{
S. Willför, R. Sjöholm, C. Laine, B. Holmbom
}

Abstract Isolated water-soluble acidic arabinogalactans from Norway spruce and Scots pine heartwood were analysed and compared to Siberian larch heartwood arabinogalactans. The carbohydrate monomer composition was determined by acid methanolysis and gas chromatography, while structural studies were performed by ${ }^{13} \mathrm{C}$ NMR spectroscopy and methylation analysis. The main structural features were found to be the same in the three types of arabinogalactans. However, the structure of the arabinogalactans from spruce and pine were found slightly different from the structure of larch arabinogalactans. The amount of single unit side-chains, consisting of arabinose and glucuronic acid units, was higher in the spruce and pine arabinogalactans than in the larch arabinogalactans. The amount of glucuronic acid was higher in the spruce arabinogalactans than in the pine arabinogalactans. The pine arabinogalactans had a higher amount of side chains with more than two sugar units than the spruce arabinogalactans.

\section{Introduction}

Water-soluble polysaccharides are released and accumulated into process waters in the production of mechanical pulp and wood-containing paper. Especially detrimental in papermaking are anionic polysaccharides, so-called "anionic trash", which can form complexes with various cationic polymers used by the

Received 30 December 1999

Stefan Willför $(\square)$, Bjarne Holmbom

Åbo Akademi University, Process Chemistry Group,

c/o Laboratory of Forest Products Chemistry,

Porthansgatan 3, 20500 Turku/Åbo, Finland

e-mail: stefan.willfor@abo.fi

Rainer Sjöholm

Åbo Akademi University, Laboratory of Organic Chemistry,

Biskopsgatan 8, 20500 Turku/Åbo, Finland

Christiane Laine

The Finnish Pulp and Paper Research Institute KCL,

P.O. Box 70, 02151 Espoo, Finland

This work is part of the activities of the Åbo Akademi Process Chemistry Group, a National Centre of Excellence supported by the Academy of Finland. The work performed at KCL was partly financed by the Technology Development Centre of Finland

(TEKES). 
paper industry. Acidic arabinogalactans (AG) constitute the main part of the anionic trash released from spruce and pine heartwood in mechanical pulping (Willför, Holmbom 1999; Willför et al. 1999; Thornton et al. 1993; 1994). The molecular structures of water-soluble arabinogalactans from different Larix species have been investigated by e.g. Ponder and Richards (1997; 1997a, b), Karácsonyi et al. (1984), Simionescu et al. (1976) and Odonmazig et al. (1994). Larch arabinogalactans consist of a main chain of $\beta$ - $\mathrm{D}$ - $(1 \rightarrow 3)$-galactopyranose units $(\beta$-D- $(1 \rightarrow 3)$-Gal $p)$ where most of the main-chain units carry a side chain on C-6 $[\rightarrow 3,6)$-Gal $p-(1 \rightarrow]$. Almost half of these side chains are $\beta$-D- $(1 \rightarrow 6)-G a l p$ dimers, and about a quarter are single Gal $p$ units. The rest contain three or more units. Arabinose is present both in the pyranose (Arap) and furanose (Araf) forms, attached to the side chains as arabinobiosyl groups [ $\beta$-L-Arap- $(1 \rightarrow 3)$-LAraf- $(1 \rightarrow]$ or as terminal $\alpha$-L-Araf. Laine et al. (1999) found 1,3-linked and partly 6 -substituted galactans in samples derived from unbleached spruce kraft pulp. The presence of glucuronic acid units in the side chains of larch arabinogalactans has not been properly addressed due to the lack of suitable analytical techniques.

In this study, the structural features of isolated water-soluble acidic arabinogalactans from the heartwood of Norway spruce and Scots pine were investigated and compared to the known structure of Siberian larch heartwood arabinogalactans. The presence of glucuronic acid units was of particular interest.

\section{Materials and methods}

\section{Isolation of water-soluble arabinogalactans from heartwood}

Stem cross sections of fresh and healthy Norway spruce, Scots pine and Siberian larch trees, grown in southern Finland, were cut and stored at $-24^{\circ} \mathrm{C}$. Knot-free parts of the heartwood, with no visible compression wood, were splintered, freeze-dried and ground in a Cyclo-Tec mill (Tecator Inc.). The wood powders were extracted in a Soxhlet apparatus with methyl tert-butyl ether (MTBE), to remove lipophilic extractives. The dry matter content of the air-dried wood powders was determined.

Portions of MTBE-extracted wood powder $(50 \mathrm{~g}$ o.d.) were suspended in 21 distilled water and the $\mathrm{pH}$ was adjusted to about 7 with $0.1 \mathrm{M} \mathrm{NaOH}$ (Willför, Holmbom 1999). The suspensions were stirred vigorously at room temperature for $1.5 \mathrm{~h}$ with a Vibro-mixer (Chemap AG). These mild extraction conditions were used in order to obtain a selective dissolution of the easily soluble arabinogalactans. The suspensions were vacuum-filtered on a GF 50 glass fiber filter (Schleicher \& Schuell). The supernatants were concentrated by vacuum evaporation using a water bath at $40{ }^{\circ} \mathrm{C}$. The concentrated supernatants were then added to technical grade ethanol, the volume percentage ethanol being at least 90 , and the polysaccharides were allowed to precipitate overnight. The samples were centrifuged and the supernatants were carefully pipetted off. The precipitates (Spruce AG, Pine AG, Larch AG) were washed with ethanol and MTBE before they were air-, and vacuum-dried.

\section{Isolation of galactoglucomannans from TMP}

Extracted TMP (hexane:acetone 9:1) from Norway spruce was suspended in distilled water at $2 \%$ consistency (Sundberg et al. 1999). The suspension was stirred with a blade propeller at about $200 \mathrm{~min}^{-1}$ and $60{ }^{\circ} \mathrm{C}$ for $3 \mathrm{~h}$. The suspension was then filtered on a paper machine wire. The TMP was again suspended in distilled water, stirred as above and filtered. The filtrates from the 
first and second stirrings were mixed and centrifuged at $500 \mathrm{~g}$ for $30 \mathrm{~min}$. The supernatant was collected and concentrated by vacuum evaporation using a water bath at $40{ }^{\circ} \mathrm{C}$. The concentrate was filtered on a Polycap ${ }^{\mathrm{TM}} 75 \mathrm{AS}$ filter obtained from Whatman with a $0.2 \mu \mathrm{m}$ pore size to remove colloidal substances. Ethanol was added to the filtrate, the volume percentage of ethanol being at least 80 . The polysaccharides were allowed to precipitate. The precipitated polysaccharides were collected and washed twice with ethanol, twice with methanol and once with MTBE. The precipitate, mainly composed of crude acetyl-galactoglucomannans (GM), was finally dried in a vacuum drier.

\section{Analyses}

Carbohydrates were analysed by acid methanolysis followed by gas chromatography (GC), according to Sundberg et al. (1996), to determine the amount and sugar composition of hemicelluloses.

${ }^{13} \mathrm{C}$ NMR spectroscopic analysis. The dried precipitates were dissolved in $\mathrm{D}_{2} \mathrm{O}$, using a short ultrasonic treatment. Acetone was added as an internal standard and given a shift of $31.5 \mathrm{ppm}$ (van Hazendonk et al. 1996). ${ }^{13} \mathrm{C}$ NMR spectra were recorded at $323 \mathrm{~K}$ with a JEOL JNM-A500 NMR spectrometer, operating at $125 \mathrm{MHz}$. An inverse gated pulse sequence was used to suppress the nOe enhancement. The pulse angle was $45^{\circ}$ and the pulse interval ca. $6 \mathrm{~s}$. These parameters facilitated the use of the signal intensities for the determination of the relative amounts of different carbon atoms in the samples, and a rough estimation of the relative amounts of the units building up the polysaccharides could be made.

Methylation analysis was performed using a modification of the method of Ciucane and Kerek (Ciucane and Kerek 1984, Laine et al. in preparation). About $20 \mathrm{mg}$ ground sodium hydroxide and $100 \mu \mathrm{l}$ methyl iodide were added to 3-5 mg sample in $500 \mu \mathrm{l}$ dimethylsulfoxide (DMSO). The sample was kept for $30 \mathrm{~min}$ in an ultrasonic bath at room temperature. Distilled water was added to the sample and the water phase was extracted with dichloromethane. The organic phase was extracted three times with distilled water, dried and evaporated. After the methylation, acid methanolysis was performed as described by Sundberg et al. (1996). After that the samples were silylated with $250 \mu \mathrm{l} \mathrm{N,O-bis(trimethyl-}$ silyl)trifluoroacetamide containing 5\% trimethylchlorosilane and the samples were analysed by GC/MS. About $1 \mu \mathrm{l}$ of silylated sample was injected via a split injector (HP7863 Series Injector/Autosampler, $260^{\circ} \mathrm{C}$, split ratio 1:50) into a $30 \mathrm{~m} / 0.25 \mathrm{~mm}$ HP-5 column (film thickness $0.25 \mu \mathrm{m}$ ) in an HP6890 Series GC System with an HP5973 Series Mass Selective Detector. The temperature program was $100{ }^{\circ} \mathrm{C}(2 \mathrm{~min})-4{ }^{\circ} \mathrm{C} / \mathrm{min}-220{ }^{\circ} \mathrm{C}(2 \mathrm{~min})-15{ }^{\circ} \mathrm{C} / \mathrm{min}-300{ }^{\circ} \mathrm{C}(2 \mathrm{~min})$. The carrier gas was helium $(1 \mathrm{ml} / \mathrm{min}$, constant flow). The detector conditions were $70 \mathrm{eV}, 40-600 \mathrm{amu}$. The retention times and mass spectra of the methylated and/or silylated monosaccharides were confirmed by comparison with earlier results of model compounds (Laine et al., in preparation).

Response factors of the permethylated and partly silylated monosaccharides were calculated based on molar responses for flame ionisation detection (Verhaar and Wilt 1969). The ratios of molar responses for mass detector and flame ionisation detector were determined experimentally and mean values of 1.40 for permethylated monosaccharides and 1.1 for partly silylated monosaccharides related to sorbitol were applied (Laine et al., in preparation). 
Table 1. Weight-\% of carbohydrate sugar units in the four studied samples as determined by acid methanolysis and GC

\begin{tabular}{llllccl}
\hline & Gal & Ara & GlcA & Man & Glc & Other \\
\hline Spruce AG & 35 & 11 & 8 & 30 & 8 & 8 \\
Pine AG & 69 & 14 & 4 & 7 & 3 & 3 \\
Larch AG & 79 & 16 & 1 & 1 & 1 & 1 \\
GM & 16 & 4 & 2 & 54 & 20 & 3 \\
\hline
\end{tabular}
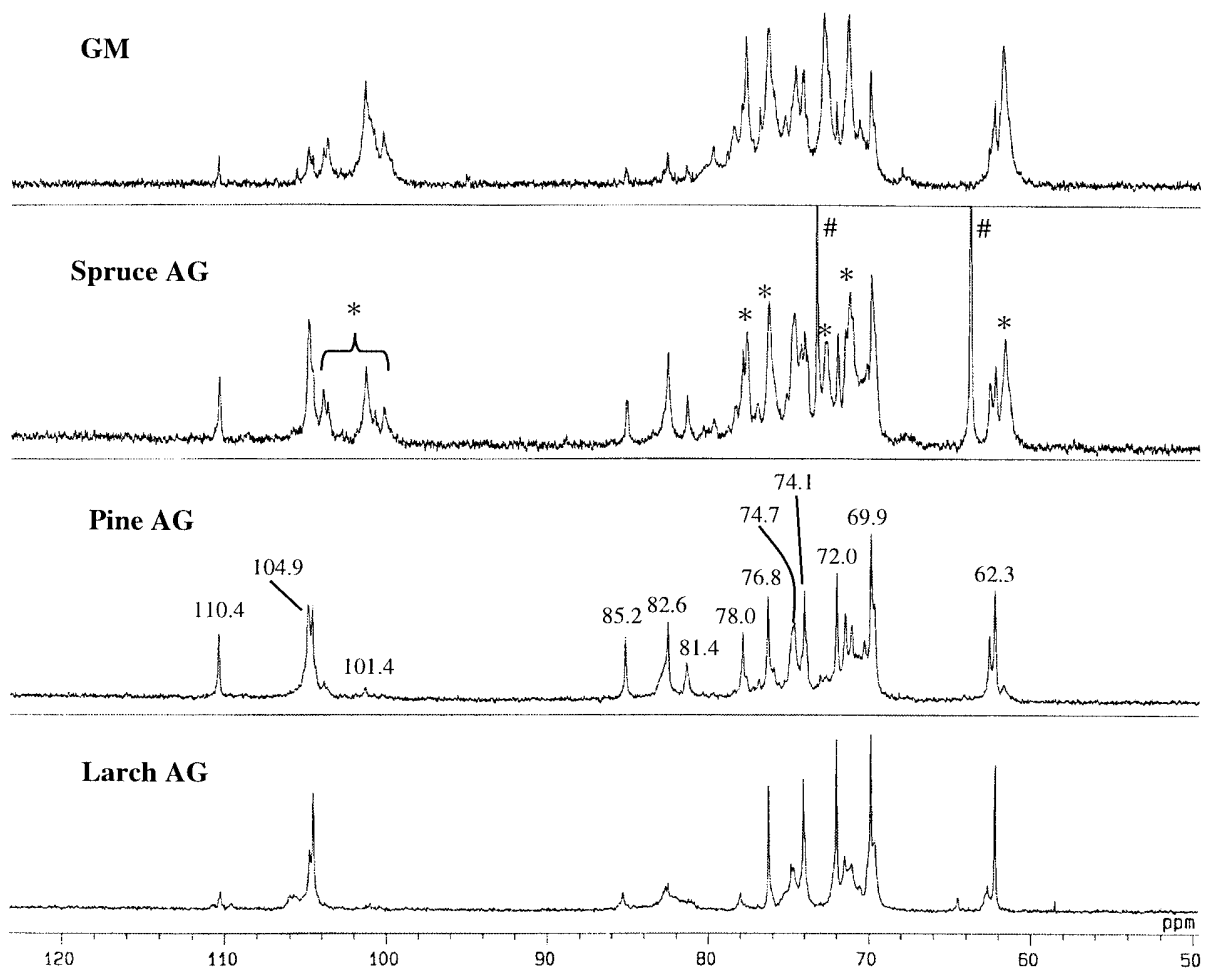

Fig. 1. Quantitative mode ${ }^{13} \mathrm{C}$ NMR spectra of spruce acetyl-galactoglucomannans (GM), spruce (Spruce AG), pine (Pine AG) and larch (Larch AG) arabinogalactans at $50{ }^{\circ} \mathrm{C} . \mathrm{D}_{2} \mathrm{O}$ was used as solvent and acetone as an internal standard $(\delta=31.5 \mathrm{ppm})$. In the spectrum of Spruce AG, the signals, which are mainly due to glucomannans, are marked with an asterisk *. Unidentified impurities in the spectrum of Spruce AG are marked with \#

\section{Results and discussion}

Acid methanolysis and GC showed that the Spruce AG sample contained both acidic arabinogalactans and neutral galactoglucomannans, while the Pine AG sample and the Larch AG sample contained mainly acidic arabinogalactans (Table 1). This was also found in an earlier study (Willför and Holmbom 1999). The GM sample isolated from spruce TMP was analysed and found to contain galactoglucomannans and a small amount of arabinogalactans.

${ }^{13} \mathrm{C}$ NMR spectra are shown in Fig. 1. The signals supposed to be derived mainly from GM are marked with an asterisk in the spectrum of Spruce AG. The chemical shifts and signal assignments for the arabinogalactans are given in 
Table $2 .{ }^{13} \mathrm{C}$ chemical shifts of the most significant signals in the spectra of the arabinogalactans

\begin{tabular}{|c|c|c|c|c|c|c|}
\hline \multirow[t]{2}{*}{ Glycosidic linkage } & \multicolumn{6}{|c|}{ Chemical shifts in $\mathrm{ppm}^{\mathrm{a}}$} \\
\hline & $\mathrm{C}-1$ & $\mathrm{C}-2$ & $\mathrm{C}-3$ & $\mathrm{C}-4$ & C-5 & $\mathrm{C}-6$ \\
\hline$\beta$-D-Galp- $(1 \rightarrow$ & 104.6 & 72.0 & 74.1 & 69.9 & 76.4 & 62.3 \\
\hline$\rightarrow 6)-\beta$-D-Galp- $(1 \rightarrow$ & 104.6 & 72.0 & 73.9 & 69.9 & 74.7 & 70.3 \\
\hline$\rightarrow 3,6)-\beta$-D-Galp- $(1 \rightarrow$ & 104.9 & 71.6 & 82.6 & 69.9 & 74.7 & 71.1 \\
\hline$\alpha$-L-Araf- $(1 \rightarrow$ & 110.4 & 81.4 & 78.0 & 85.2 & 62.6 & \\
\hline$\beta$-L-Arap- $(1 \rightarrow$ & 101.4 & 69.7 & ${ }_{-}^{b}$ & $\mathrm{~b}^{\mathrm{b}}$ & $63.9^{\mathrm{c}}, 64.6^{\mathrm{d}}$ & \\
\hline$\rightarrow 3)-\alpha$-L-Araf- $(1 \rightarrow$ & $108.6^{\mathrm{d}}$ & $-\mathrm{b}$ & $-{ }^{b}$ & ${ }^{\mathrm{b}}$ & - b & \\
\hline
\end{tabular}

${ }^{\mathrm{a}}$ Relative to internal acetone $(\delta=31.5 \mathrm{ppm})$;

${ }^{\mathrm{b}}$ Not detected;

${ }^{\mathrm{c}}$ In the spectrum of Pine AG;

${ }^{\mathrm{d}}$ In the spectrum of Larch AG

Table 3. Mole- $\%$ of carbohydrate monomers as determined by methylation analysis; $\mathrm{T}=$ terminal non-reducing end unit

\begin{tabular}{lrrrrrrrrr}
\hline & Gal & & \multicolumn{1}{c}{ Ara } & Man & & \multicolumn{1}{c}{ Glc } \\
& \multicolumn{1}{c}{ T } & 1,3 & 1,6 & $1,3,6$ & \multicolumn{1}{c}{ T } & \multicolumn{1}{c}{ T } & 1,4 & $1,4,6$ & 1,4 \\
\hline Spruce AG & 7.1 & 1.4 & 1.2 & 3.7 & 3.1 & 2.0 & 61.5 & 4.6 & 15.3 \\
Pine AG & 26.0 & 8.3 & 6.9 & 23.9 & 17.3 & & 13.2 & 1.0 & 3.5 \\
Larch AG & 36.8 & 2.3 & 14.6 & 26.6 & 19.7 & & & & \\
\hline
\end{tabular}

Table 2. The assignments were based on the data published by Ponder et al. (1997a) on arabinogalactans isolated from Western larch (Larix occidentalis).

The three arabinogalactan samples were also analysed by methylation analysis and the results are given in mole percent in Table 3 . The quantification gives approximate ratios between the structural elements in one sample. We experienced some solubility problems of the arabinogalactans in the alkaline solvent (DMSO) during the methylation. Consequently, the results may not be fully representative for the sample as a whole. We cannot exclude that the structure of the DMSO-soluble fraction of the arabinogalactans differs from the $\mathrm{D}_{2} \mathrm{O}$-soluble fraction analysed by ${ }^{13} \mathrm{C}$ NMR spectroscopy. The methylation analysis did not give information about acidic groups, which were not identified in the gas chromatograms.

\section{Galactose units}

The ${ }^{13} \mathrm{C}$ NMR results showed that the water-soluble arabinogalactans all have a main chain consisting of $\beta$-D- $(1 \rightarrow 3)$-Gal $p$-units. The main-chain units are mainly $(1 \rightarrow 6)$-linked to side chains of galactose, arabinose or glucuronic acid units. The Galp monomer unit ratios, based on the ${ }^{13} \mathrm{C}$ NMR analysis, in the three AG-samples are given in Table 4 . The ratios of the signals at $\delta=82.6 \mathrm{ppm}[\rightarrow 3,6)$ Galp- $(1 \rightarrow, \mathrm{C}-3]$ and $\delta=62.3 \mathrm{ppm}[\beta-\mathrm{D}-\mathrm{Gal} p-(1 \rightarrow, \mathrm{C}-6]$ were used for the determination of the monomer unit ratio $\rightarrow 3,6)-\mathrm{Gal} p-(1 \rightarrow / \mathrm{Gal} p-(1 \rightarrow$. From the signals at $\delta=102.6-106.1 \mathrm{ppm}$ the monomer unit ratio $\rightarrow 3,6)-\mathrm{Gal} p-(1 \rightarrow / \mathrm{Gal} p-$ $(1 \rightarrow+\rightarrow 6)-$ Gal $p$ - $(1 \rightarrow$ could be determined. Combining the information from these signals facilitated the determination of the ratios $\rightarrow 3,6)-\mathrm{Gal} p-(1 \rightarrow / \rightarrow 6)-\mathrm{Gal} p-(1 \rightarrow$, Gal $p-(1 \rightarrow / \rightarrow 6)$-Gal $p-(1 \rightarrow$ and Gal $p-(1 \rightarrow / \rightarrow 3,6)-\mathrm{Gal} p-(1 \rightarrow+\rightarrow 6)-\mathrm{Gal} p-(1 \rightarrow$. 
Table 4. Monomer unit ratios in the samples, based on ${ }^{13} \mathrm{C} N M R$

\begin{tabular}{llll}
\hline Ratios & \multicolumn{3}{l}{ Monomer unit ratios } \\
\cline { 2 - 4 } & Spruce AG & Pine AG & Larch AG \\
\hline$\rightarrow 3,6)$-Gal $p-(1 \rightarrow /$ Gal $p-(1 \rightarrow$ & $2.5 / 1$ & $2.1 / 1$ & $2.0 / 1$ \\
$\rightarrow 3,6)-G a l p-(1 \rightarrow / G a l p-(1 \rightarrow+\rightarrow 6)-G a l p-(1 \rightarrow$ & $2.25 / 1$ & $1.7 / 1$ & $1.4 / 1$ \\
$\rightarrow 3,6)-G a l p-(1 \rightarrow / \rightarrow 6)-G a l p-(1 \rightarrow$ & $31.1 / 1$ & $2.8 / 1$ & $20.6 / 1$ \\
Gal $p-(1 \rightarrow / \rightarrow 6)-G a l p-(1 \rightarrow$ & $12.7 / 1$ & $0.9 / 1$ & $3.9 / 1$ \\
Gal $p-(1 \rightarrow / \rightarrow 3,6)-G a l p-(1 \rightarrow+\rightarrow 6)-G a l p-(1 \rightarrow$ & $0.4 / 1$ & $0.3 / 1$ & $0.4 / 1$ \\
\hline
\end{tabular}

The monomer unit ratios suggest that almost $70 \%$ of the galactose in the Spruce AG sample is present in a main chain as $[\rightarrow 3,6)$-Galp- $(1 \rightarrow]$-linked units. For the Pine AG sample this number is about $55 \%$ and for the Larch AG sample about $65 \%$. The percentage of terminal [Galp- $(1 \rightarrow]$-units is for the Spruce AG, Pine AG and Larch AG samples $28 \%, 22 \%$ and $28 \%$, respectively. The percentage of $[\rightarrow 6)$-Gal $p-(1 \rightarrow]$-linked non-terminal side chain units is as much as $23 \%$ for the Pine AG sample, while for Spruce AG it is only $2 \%$ and for Larch AG 7\%. It is therefore likely that the Pine AG sample has longer side chains, with at least two $[\rightarrow 6)$-Galp- $(1 \rightarrow]$-linked non-terminal side chain units.

We were not able to show the presence of any unsubstituted $(1 \rightarrow 3)$-linked Galp-units by ${ }^{13} \mathrm{C}$ NMR analysis, based on comparison with NMR data published by Usov et al. (1997). However, the methylation analysis (Table 3) clearly showed the presence of such units. Slightly more than $10 \%$ of the Spruce AG and Pine AG and under $3 \%$ of the Larch AG galactose was unsubstituted $(1 \rightarrow 3)$-linked Galpunits. However, these numbers are approximate, since the yield of the methylation analysis was low, as mentioned earlier. For larch arabinogalactans, Ponder and Richards (1997b) suggested the presence of about $4 \%$ of unsubstituted $(1 \rightarrow 3)$-linked Galp-units.

\section{Arabinose units}

In all samples, arabinose was present as terminal $\alpha$-L-Araf end groups attached to the galactopyranose-backbone. Earlier it has been shown by Ponder and Richards (1997b) that arabinose is present as terminal Araf or as arabinobiosylgroups [ $\beta$-LArap-(1 $\rightarrow 3)-\alpha$-L-Araf- $(1 \rightarrow]$. According to Karácsonyi et al. (1984), Odonmazig et al. (1994) and Ponder and Richards (1997a), C-5 in Ara $p$ units absorbs at $\delta=63.8,64.5$ or $64.4 \mathrm{ppm}$, respectively. There was no signal detected for the Spruce AG sample, which could be assigned to Arap units. For the Pine AG sample there was a weak signal at $\delta=64.1 \mathrm{ppm}$ that indicated the presence of Arap units. The intensity of the signal as compared with the total of the anomeric carbons (excluding Arap) was Arap C-5/-O-C-O- $\approx 1 / 93.6$, corresponding to an amount of ca. 1\% Arap units. For the Larch AG sample there was a weak signal at $\delta=64.6 \mathrm{ppm}$ that indicated the presence of Arap units. The intensity of the signal as compared with the total of the anomeric carbons (excluding Arap) was Ara $p$ C-5/-O-C-O- $\approx 1 / 18.0$, corresponding to an amount of ca. $5 \%$ Arap units. The methylation analysis showed the presence of terminal Araf but also weak signals that could be assigned to Arap units.

The intensity ratios of the signals at $\delta=110.3 \mathrm{ppm}$ (Araf, C-1) and $\delta=102.6-$ $106.1 \mathrm{ppm}(\mathrm{Gal} p, \mathrm{C}-1)$ were used to determine the Araf/Gal $p$ ratio. The contribution of the GM signals was subtracted from the Galp signals in the Spruce and 
Pine AG samples. The calculated ratio Araf/Galp for the Spruce AG sample was found to be $\approx 0.21 / 1$. Ca. $6 \%$ of the signals from anomeric carbons remained unidentified (including Ara $p, \mathrm{C}-1$ ). The calculated ratio Araf/Gal $p$ for the Pine AG sample was found to be $\approx 0.26 / 1$. Ca. $3 \%$ of the signals from anomeric carbons remained unidentified (including Ara $p, \mathrm{C}-1$ ). The calculated ratio Araf/Gal $p$ for the Larch AG sample was found to be $\approx 0.14 / 1$. Ca. $3 \%$ of the signals from anomeric carbons remained unidentified. These numbers are well in agreement with the ones obtained from the methylation analysis, except for the Larch AG sample. The calculated ratio Araf/Galp for the Larch AG sample was $\approx 0.25 / 1$ according to the methylation analysis.

\section{Glucuronic acid units}

The amount of acidic groups (GlcA) was determined as the relative intensities of the signals of $C=\mathrm{O}(\delta=173.6-175.1 \mathrm{ppm})$ and the anomeric carbon (C-1). The sample of Spruce AG contained some GM and the spectrum contained strong signals, which could be assigned to acetyl carbons in spruce glucomannans. The intensity of the signal of $C=\mathrm{O}$ was determined by manual subtraction of the intensity of the corresponding GM signal. The contribution of GM to the carbonyl signal was estimated by first determining the intensity ratio of the signals at $\delta=97.6-102.6 \mathrm{ppm}$ and the carbonyl signal in the spectrum of GM. The ratio was found to be $2.53 / 1$. Then the intensities of the corresponding signals in the spectrum of Spruce AG were determined. In this way the ratio $C=\mathrm{O} / \mathrm{C}-1$ was found to be $\approx 0.08$, corresponding to an amount of ca. $7-8 \%$ uronic acid units. As the Pine AG sample also contained small amounts of GM, the contribution of GM to the carbonyl signal was first determined. Determined in the same way as described for the Spruce AG sample the ratio $C=\mathrm{O} / \mathrm{C}-1$ was found to be $\approx 0.02$, corresponding to an amount of ca. $2 \%$ uronic acid units. No signals from carboxyl carbons could be detected in the Larch AG sample. This was probably due to the low amount of GlcA (Table 1).

\section{Suggested major structural features for spruce and pine arabinogalactans}

The major structural conclusions in this study are drawn from the ${ }^{13} \mathrm{C} \mathrm{NMR}$ analysis. In addition, the methylation analysis and the carbohydrate monomer composition provided some important structural features: e.g the presence of unsubstituted $(1 \rightarrow 3)$-bonded Galp-units was shown by the methylation analysis and the presence of GlcA in the Larch AG sample was seen from the carbohydrate monomer composition. The amounts of these units were probably under the limit of detection for the NMR analysis.

Figure 2 shows a suggested average structure for water-soluble Norway spruce arabinogalactans. The structure is probably slightly different for different molar masses and that the mass range of these samples was quite broad (Willför and Holmbom 1999). An average molar mass of about 22 kdaltons corresponds to a degree of polymerisation (DP) of 130-140. It is therefore unlikely that the different types of side chains will all be present in one single molecule. However, a typical spruce arabinogalactan molecule has a shorter main chain than a typical larch arabinogalactan molecule. It has also more single unit side chains than larch arabinogalactan and a higher amount of these are acidic. The low amount of $[\rightarrow 6)-G a l p-(1 \rightarrow]$-units suggests that the probability of side chains with more than two units is very low.

Figure 3 shows a suggested average structure for water-soluble Scots pine arabinogalactans. As mentioned above, the DP (190-200) and broad mass range 


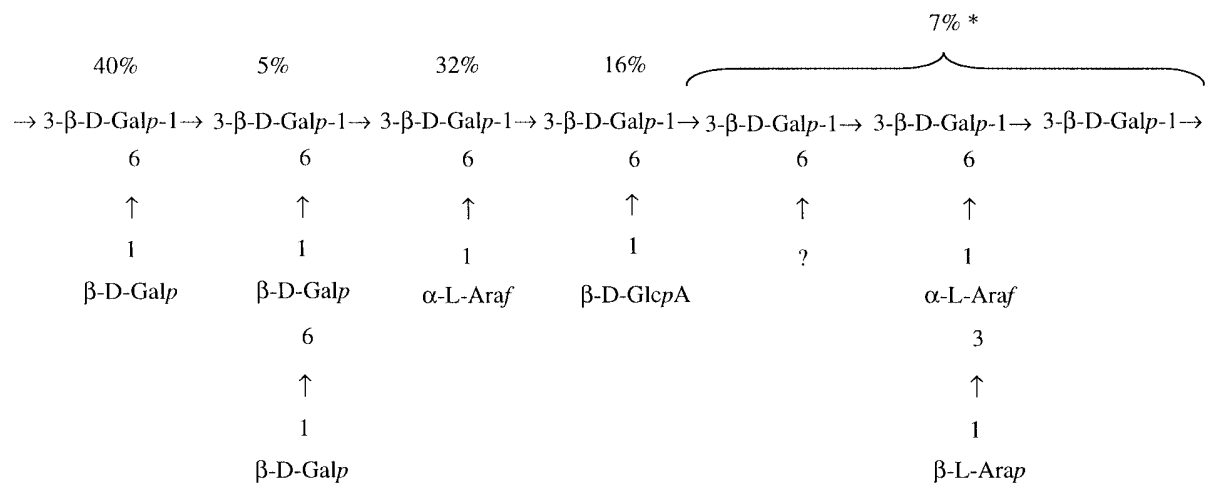

Fig. 2. Suggested major structural features of a typical spruce arabinogalactan molecule. The probability and ratio of the different side chains probably varies with the molar mass and between different molecules. ${ }^{*}$ The ${ }^{13} \mathrm{C}$ NMR analysis did not show the presence of any arabinobiosyl groups or unsubstituted galactose units in the main chain. The presence of these groups was shown by the methylation analysis. About $7 \%$ of the sugar units remain undefined

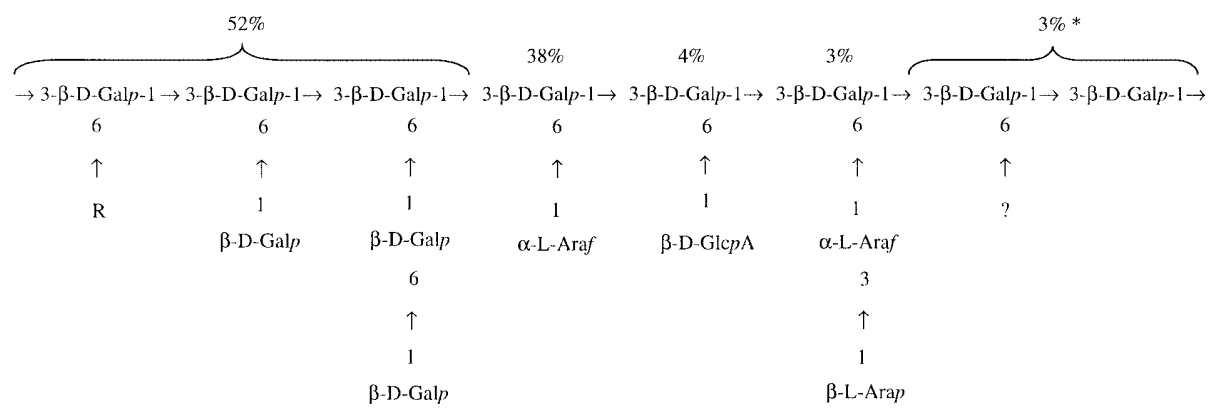

Fig. 3. Suggested major structural features of a typical pine arabinogalactan molecule. The probability and ratio of the different side chains probably varies with the molar mass and between different molecules. ${ }^{*}$ The ${ }^{13} \mathrm{C}$ NMR analysis did not show the presence of any unsubstituted galactose units in the main chain. The presence of these groups was shown by the methylation analysis. About $3 \%$ of the sugar units remain undefined. $\mathrm{R}=$ sidechains containing three or more $\beta$-D-Gal $p$ unit

Table 5. Molar sugar unit ratios of the isolated AG samples determined with the different analytical techniques

\begin{tabular}{llll}
\hline & $\begin{array}{l}\text { Acid methanolysis and GC } \\
\text { Gal:Ara:GlcA }\end{array}$ & $\begin{array}{l}\text { Methylation analysis } \\
\text { Gal:Ara }\end{array}$ & $\begin{array}{l}{ }^{13} \mathrm{C} \mathrm{NMR} \\
\text { Gal:Ara:GlcA }\end{array}$ \\
\hline Spruce AG & $2.6: 1: 0.6$ & $2.8: 1$ & $4.8: 1: 0.5$ \\
Pine AG & $3.9: 1: 0.2$ & $3.7: 1$ & $3.7: 1: 0.1$ \\
Larch AG & $4.1: 1: 0.04$ & $4.1: 1$ & $5.0: 1:-$ \\
\hline
\end{tabular}

suggest a variable side chain structure for single molecules. The typical pine arabinogalactan molecule is probably slightly shorter than the spruce molecule. There are less acidic side chains and the relatively high amount of $[\rightarrow 6)-G a l p-$ 
$(1 \rightarrow]$-units suggests that the probability of side chains with more than two units is high. It was not possible to suggest the ratio of the three Galp-type side chains.

\section{Sugar unit ratios}

The sugar unit ratios of the isolated AG samples determined by acid methanolysis and GC, methylation analysis, and ${ }^{13} \mathrm{C}$ NMR spectroscopy were in good agreement (Table 5). The Gal:Ara ratio for Spruce AG, which was determined by ${ }^{13} \mathrm{C}$ NMR, was high. This may be explained by the unidentified signals from the anomeric carbons. Some arabinogalactans were lost during the isolation procedure.

Therefore, it is likely that the previously reported ratios of 3.6:1:0.8 for spruce, 3.8:1:0.2 for pine, and 5.6:1:0.08 for larch are more representative for the total water-soluble arabinogalactans (Willför, Holmbom 1999).

\section{References}

Ciucane I, Kerek F (1984) A simple and rapid method for the permethylation of carbohydrates. Carbohydr Res 131: 209-217

van Hazendonk JM, Reinerink EJM, de Waard P, van Dam JEG (1996) Structural analysis of acetylated polysaccharides from fibre flax (Linum usitatissimum L.). Carbohydr Res 291: 141-154

Karácsonyi S, Kovácik V, Alföldi J, Kubacková M (1984) Chemical and ${ }^{13}$ C-N.M.R. studies of an arabinogalactan from Larix sibirica L. Carbohydr Res 134:265-274

Laine C, Haakana S, Hortling B, Tamminen T (1999) Application of methylation analysis to samples derived from spruce kraft pulp. 10th Intl. Symp. Wood Pulping Chem., Vol. 1, Japan Tappi 72-77

Laine C, et al. unpublished results in preparation

Ponder GR, Richards GN (1997) Arabinogalactan from Western larch, Part I; effect of uronic acid groups on size exclusion chromatography. J Carbohydr Chem 16:181-193

Ponder GR, Richards GN (1997a) Arabinogalactan from Western larch, Part II; a reversible order-disorder transition. J Carbohydr Chem 16:195-211

Odonmazig P, Ebringerova A, Machová E, Alföldi J (1994) Structural and molecular properties of the arabinogalactan isolated from Mongolian larchwood (Larix dahurica L.). Carbohydr Res 254:317-324

Ponder GR, Richards GN (1997b) Arabinogalactan from Western larch, Part III; alkaline degradation revisited, with novel conclusions on molecular structure. Carbohydr Polym 34:251-261

Simionescu C, Sang Il B, Cernatescu-Asandei A (1976) Researches in the field of chemistry and technology of larch wood pulping by magnesium bisulphite process. II. Structure of arabinogalactan from larch wood (Larix decidua Mill). Cellulose Chem Technol 10:535-545

Sundberg A, Sundberg K, Lillandt C, Holmbom B (1996) Determination of hemicelluloses and pectins in wood and pulp fibres by acid methanolysis and gas chromatography. Nord. Pulp Pap Res J 4:216-219, 226

Sundberg A, Holmbom B, Willför S, Pranovich A (1999) Weakening of paper strength by wood resin. Accepted for publication in Nord Pulp Pap Res J

Timell TE (1986) In "Compression wood in gymnosperms" Springer-Verlag, Berlin 1:353$359,432-433$

Thornton J, Ekman R, Holmbom B, Eckerman C (1993) Release of potential “anionic trash" in peroxide bleaching of mechanical pulp. Pap Puu 75:426-431

Thornton J, Ekman R, Holmbom B, Örså F (1994) Polysaccharides dissolved from Norway spruce in thermomechanical pulping and peroxide bleaching. J Wood Chem Technol 14:159-175

Usov AI, Bilan MI, Shashkov AS (1997) Structure of a sulfated xylogalactan from the calcareous red algae Corallina pilulifera P. et R. (Rhodophyta, Corallinaceae). Carbohydr Res 303:93-102 
Verhaar LATh, De Wilt HGJ (1969) The gas chromatographic determination of polyhydroxy monocarbonic acids obtained by oxygenation of hexoses in aqueous alkaline solutions. J Chromatog 41:168-179

Willför S, Holmbom B (1999) Structural features of water-soluble arabinogalactans from Norway Spruce and Scots pine heartwood. Wood Sci Technol DOI 10.1007/s00226-0010137-x (this issue)

Willför S, Sjöholm R, Holmbom B (1999) Isolation and characterisation of water-soluble arabinogalactans from the heartwood of Norway spruce and Scots pine. 10th Intl. Symp., Japan Tappi. Wood Pulping Chem 2:32-34 